\title{
Deconfinement of heavy quarks at finite density and strong magnetic field
}

\author{
Michał Szymański ${ }^{\mathrm{a}}$ \\ Institute of Theoretical Physics, University of Wrocław, plac Maksa Borna 9, \\ 50-204 Wrocław, Poland
}

Received 13 June 2020 / Accepted 27 October 2020

Published online 21 December 2020

\begin{abstract}
We study the simultaneous effect of the external magnetic field and finite chemical potential on deconfinement of heavy quarks, probed by the Polyakov loop and its fluctuations. We calculate the Polyakov loop, its real and imaginary susceptibilities, their ratio, as well as the heavy quark and anti-quark entropies. We find that these quantities are sensitive probes of deconfinement. Especially, the real Polyakov loop susceptibility and heavy quark and anti-quark entropies diverge at the critical point.
\end{abstract}

\section{Introduction}

Understanding the deconfinement is one of the long-standing goals of high-energy physics, relevant for the proper interpretation of experimental data on relativistic heavy-ion collisions [1]. Especially important in this context is studying the impact of the baryon chemical potential [2] and external magnetic field [3] on the transition, as well as its relation to chiral symmetry restoration.

Deconfinement is best understood in the pure Yang-Mills theory, where it can be related to the spontaneous breaking of the $Z_{3}$ center symmetry $[4,5]$. As an order parameter related to that transition one usually considers the Polyakov loop [6-8] which measures the free energy of a static color source immersed in a hot medium. Its expectation value vanishes below the critical temperature which corresponds to infinite energy of the static quark and is interpreted as confinement. In this case, the system is invariant under the center symmetry. Above the critical temperature, Polyakov loop expectation value is greater than zero and the center symmetry is spontaneously broken. For three colors the transition is first-order.

When dynamical quarks are present in the system, the $Z_{3}$ symmetry becomes broken explicitly. For the sufficiently large breaking, deconfinement can turn into the second-order transition and then into a crossover. In the last case, the Polyakov loop is no longer a true order parameter but nevertheless it remains a useful probe of the screening properties of QCD medium [9]. Through the coupling to quarks, deconfinement becomes also sensitive to external parameters, including finite chemical potential [10-13] and magnetic field [14-16].

a e-mail: michal.szymanski@uwr.edu.pl 
Susceptibilities of Polyakov loop provide another set of observables relevant for studying deconfinement [17]. These quantities are discontinuous at the first-order transition and have a peak in case of the crossover. The last property provides a way to define (a non-unique) crossover temperature. Another observable sensitive to deconfinement is the ratio of transverse (imaginary) to longitudinal (real) susceptibilities. In pure gauge theory, this quantity exhibits a step-function behavior $[17,18]$ and in the presence of light dynamical quarks it becomes considerably smoothened but remains sensitive to deconfinement $[18,19]$. It is also useful as it provides means to quantify the strength of explicit center symmetry breaking [20]. Another interesting observable is the heavy quark entropy, defined as the temperature derivative of the heavy quark free energy. It is sensitive to deconfinement and was argued to provide a more accurate way to define the critical temperature of deconfinement than the Polyakov loop inflection point when calculated on the lattice [19].

In this work, we discuss the simultaneous effect of the strong magnetic field and finite chemical potential on the Polyakov loop and its fluctuations in the presence of heavy quarks. This allows to omit difficulties arising from the chiral aspects of QCD and focus only on the deconfinement. Particularly, we extend previous studies $[13,16]$, where the impact of finite chemical potential and external magnetic field on the deconfinement was investigated separately. The article is organized as follows: In Section 2 we introduce the model and define various observables which are considered in the current work. Section 3 comprises the discussion of numerical results obtained using the model and in Section 4 we conclude our paper.

\section{Polyakov loop model}

To study the impact of the external magnetic field and finite chemical potential on the deconfinement we model the effective QCD potential as

$$
\mathcal{U}=\mathcal{U}_{G}+\mathcal{U}_{Q}
$$

where $\mathcal{U}_{G}$ is the pure gauge Polyakov loop potential and $\mathcal{U}_{Q}$ contains the contribution due to dynamical quarks. The pure gauge part is invariant under center symmetry transformations and exhibits its spontaneous breaking. The quark contribution breaks the $Z_{3}$ symmetry explicitly. In the current study, we use the following Polyakov loop potential [18],

$$
\frac{\mathcal{U}_{G}}{T^{4}}=-\frac{A}{2} L \bar{L}+B \ln M_{H}(L, \bar{L})+\frac{C}{2}\left(L^{3}+\bar{L}^{3}\right)+D(L \bar{L})^{2},
$$

where $L$ and $\bar{L}$ are Polyakov loop and its conjugate, $M_{H}(L, \bar{L})$ is the $\mathrm{SU}(3)$ Haar measure,

$$
M_{H}(L, \bar{L})=1-6 L \bar{L}+4\left(L^{3}+\bar{L}^{3}\right)-3(L \bar{L})^{2}
$$

and $A, B, C$ and $D$ are temperature-dependent model parameters which were determined from the lattice data on the pure gauge equation of state, renormalized Polyakov loop and its fluctuations [18].

To incorporate the effect of dynamical quarks in the model we use the one-loop effective potential of quarks interacting with the uniform background of temporal gluon field. We consider only the single-flavor case since the presence of additional flavors does not change the qualitative aspects of our results. In the absence of the 
external magnetic field, the fermion contribution reads

$$
\mathcal{U}_{Q}(L, \bar{L}, T, \mu)=-2 T \int \frac{d^{3} p}{(2 \pi)^{3}}\left(\ln g_{f}^{+}+\ln g_{f}^{-}\right)
$$

where

$$
\begin{aligned}
& g_{f}^{+}=1+3 L e^{-\beta(E-\mu)}+3 \bar{L} e^{-2 \beta(E-\mu)}+e^{-3 \beta(E-\mu)}, \\
& g_{f}^{-}=1+3 \bar{L} e^{-\beta(E+\mu)}+3 L e^{-2 \beta(E+\mu)}+e^{-3 \beta(E+\mu)},
\end{aligned}
$$

with $\mu$ the chemical potential and $E^{2}=\mathbf{p}^{2}+m^{2}$, where $m$ is the quark mass.

To study the impact of the external magnetic field on the model thermodynamics, we consider a constant, homogeneous background magnetic field, pointing in the $z$ direction. In such conditions, the motion of charged particles undergoes the Landau quantization in the transverse plane. Consequently, the dispersion relation becomes modified and takes the following form for spin $1 / 2$ particles,

$$
E_{k, \sigma}^{2}=m^{2}+p_{z}^{2}+(2 k+1-\sigma)|q B|
$$

where the subsequent Landau levels are quantified by $k=\{0,1,2 \ldots\}$ and $\sigma= \pm 1$ (the spin projection on the $\mathbf{B}$ axis). The sum over states is modified accordingly,

$$
2 \int \frac{d^{3} p}{(2 \pi)^{3}} \rightarrow \frac{|q B|}{2 \pi} \sum_{\sigma= \pm 1} \sum_{k=0}^{\infty} \int_{-\infty}^{\infty} \frac{d p_{z}}{2 \pi}
$$

where the summation runs over Landau levels and the factor $|q B| /(2 \pi)$ accounts for the planar density of each Landau level. Therefore, the quark contribution at finite magnetic field reads

$$
\mathcal{U}_{Q}(L, \bar{L}, T, \mu, q B)=-\frac{T|q B|}{2 \pi} \sum_{\sigma= \pm 1} \sum_{k=0}^{\infty} \int_{-\infty}^{\infty} \frac{d p_{z}}{2 \pi}\left(\ln g_{f}^{+}+\ln g_{f}^{-}\right)
$$

When the magnetic field vanishes, this equation agrees with equation (4).

Through this work we use the mean-field approximation. To obtain expectation values of Polyakov loop and its conjugate we solve the gap equations,

$$
\frac{\partial \mathcal{U}}{\partial L}=0, \quad \frac{\partial \mathcal{U}}{\partial \bar{L}}=0 .
$$

In this approximation, susceptibilities of Polyakov loop are obtained from the inverse of the curvature matrix,

$$
T^{3} \chi=\mathcal{C}^{-1}
$$

where

$$
\mathcal{C}=\frac{1}{T^{4}}\left(\begin{array}{cc}
\frac{\partial^{2} \mathcal{U}}{\partial L^{2}} & \frac{\partial^{2} \mathcal{U}}{\partial L \partial L} \\
\frac{\partial^{2} \mathcal{U}}{\partial L \partial L} & \frac{\partial^{2} \mathcal{U}}{\partial L^{2}}
\end{array}\right)
$$


It is evaluated at $\left(L_{0}, \bar{L}_{0}\right)$ obtained by solving the gap equations (10). In this work we consider fluctuations of the real and imaginary parts of Polyakov loop which are connected to $\chi_{L L}, \chi_{\bar{L} \bar{L}}$ and $\chi_{L \bar{L}}$ by the following relations [22],

$$
\begin{aligned}
\chi_{L} & =\frac{1}{2} \chi_{L \bar{L}}+\frac{1}{4}\left(\chi_{L L}+\chi_{\bar{L} \bar{L}}\right), \\
\chi_{T} & =\frac{1}{2} \chi_{L \bar{L}}-\frac{1}{4}\left(\chi_{L L}+\chi_{\bar{L} \bar{L}}\right) .
\end{aligned}
$$

The following ratio can be formed from these susceptibilities $[17,18]$,

$$
R_{T}=\chi_{T} / \chi_{L}
$$

which was found to be a sensitive probe of the explicit $Z_{3}$ symmetry breaking. Another observable relevant for the deconfinement is the heavy quark entropy, defined as the temperature derivative of the heavy quark free energy, which in terms of Polyakov loop can be expressed as

$$
S_{Q}=\frac{\partial}{\partial T} T \ln \langle L\rangle
$$

Similarly, the heavy anti-quark entropy

$$
S_{\bar{Q}}=\frac{\partial}{\partial T} T \ln \langle\bar{L}\rangle
$$

can be defined. Heavy quark entropy was argued to provide a scheme-independent way of defining the critical temperature of deconfinement from tracking its peak [19].

\section{Numerical results}

An important feature of first-order phase transitions is the stability under small explicit symmetry breaking [21]. Therefore, for three colors the deconfinement remains first-order unless the critical value of breaking due to the presence of dynamical quarks is reached. For the model considered in this work, the critical value of the quark mass needed to reach the deconfinement critical point in the absence of other external parameters is

$$
m_{0}=1.1 \mathrm{GeV}
$$

for one quark flavor [13].

The left column of Figure 1 shows the results on Polyakov loop (thick colored lines) and its conjugate (thin gray lines) calculated for quark mass $1.4 m_{0}=1.54 \mathrm{GeV}$. Since the mass is above the critical value, the deconfinement in the absence of other parameters is first-order, which is reflected in the discontinuity of the Polyakov loop (the solid blue and gray lines). For the vanishing chemical potential Polyakov loop and its conjugate are equal (the upper row). The external magnetic field enhances the $Z_{3}$ breaking strength and for its sufficiently large value, the deconfinement reaches the critical point (the dashed red and gray lines) and, for even larger values of $q B$, the crossover (the dash-dotted green and gray lines).

In the presence of finite chemical potential the Polyakov loop and its conjugate obtained from solving the gap equations are real but in general different. This is 

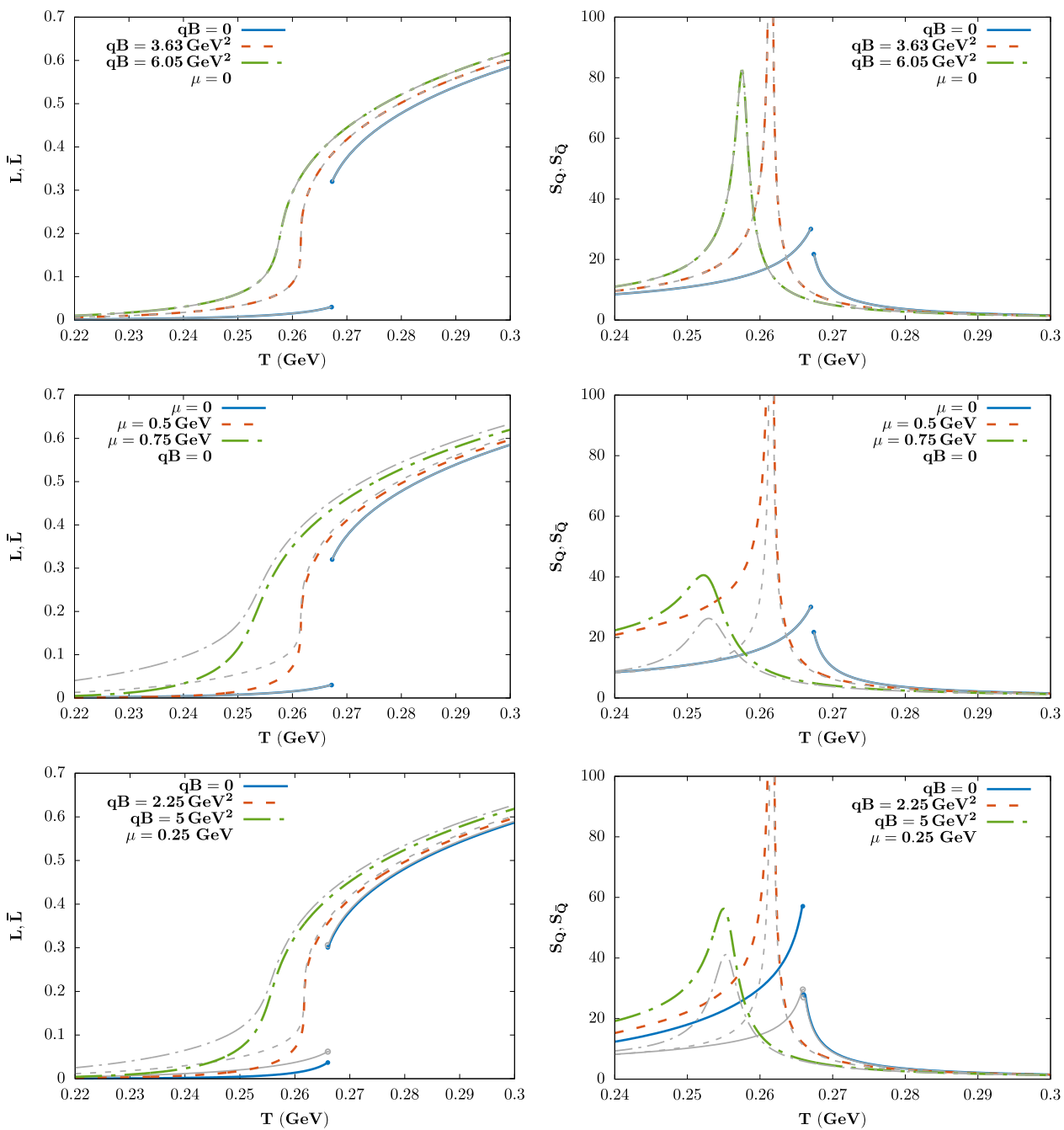

Fig. 1. Left column: Polyakov loop expectation value (thick colored lines) and its conjugate (thin gray lines) calculated for various values of the magnetic field and chemical potential. Right column: The corresponding heavy quark (thick colored lines) and anti-quark (thin gray lines) entropies. For all figures, the quark mass is $1.4 m_{0}=1.54 \mathrm{GeV}$.

because in this case the action is complex $[9,22,23]$. The temperature dependence of the Polyakov loop and its conjugate at vanishing magnetic field is shown on the left middle panel of Figure 1. At $\mu=0.5 \mathrm{GeV}$, the deconfinement transition becomes second order and the turns into a crossover. Therefore, the finite chemical potential also increases the strength of the explicit $Z_{3}$ symmetry breaking.

Finally, the lower left panel shows a simultaneous contribution of the finite chemical potential, $\mu=0.25 \mathrm{GeV}$, and external magnetic field, $q B=0,2.25$ and $5 \mathrm{GeV}^{2}$ (the solid, dashed and dash-dotted lines). In this case, the magnetic field needed to turn deconfinement into second-order phase transition is lower than in the $\mu=0$ case, which is because both quantities enhance the strength of $Z_{3}$ symmetry breaking.

Corresponding fluctuations can be seen on Figure 2, where the left column corresponds to the longitudinal susceptibility and the right column to the transverse 

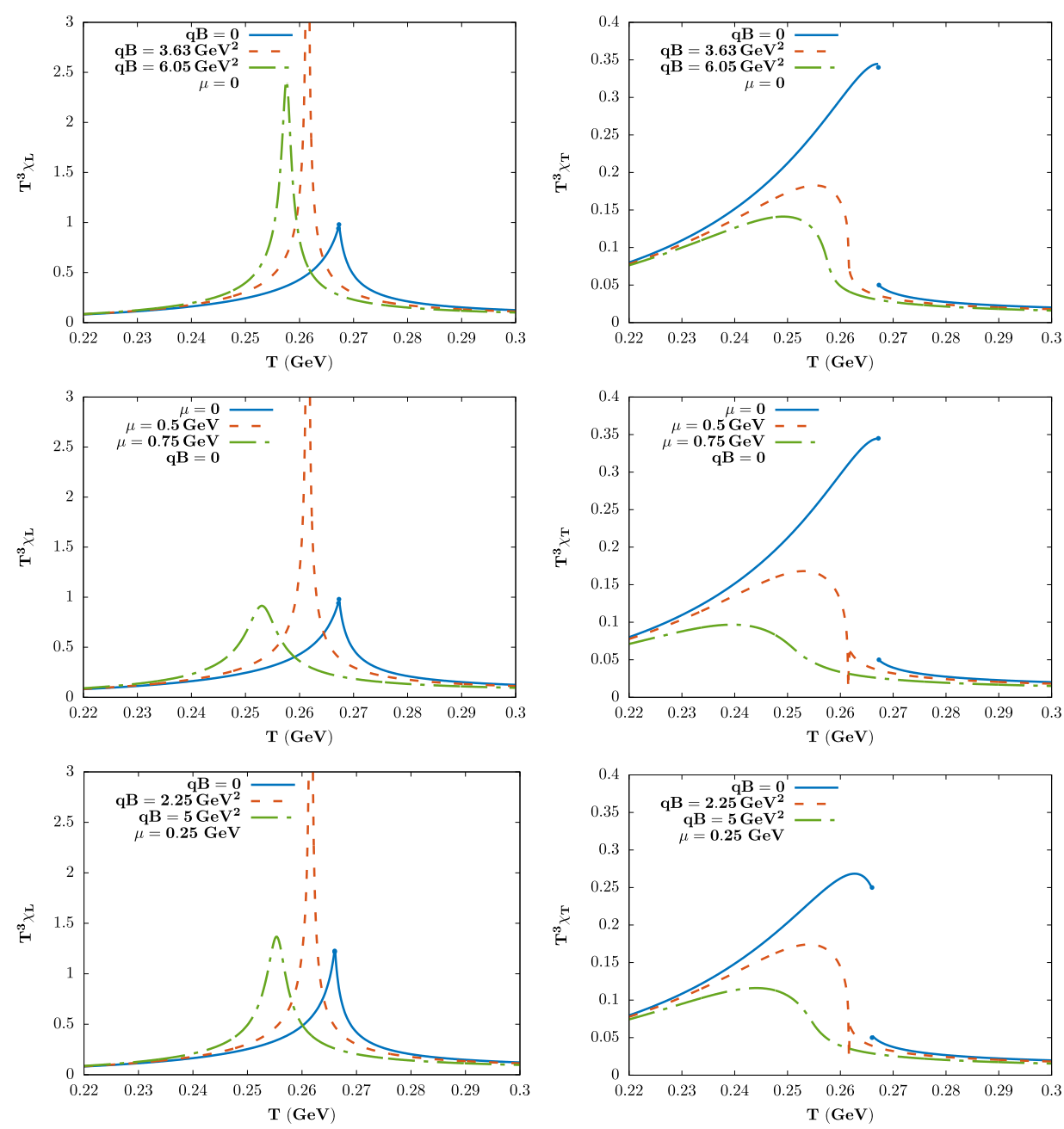

Fig. 2. Left column: The longitudinal (real) susceptibility of the Polyakov loop calculated for various values of the magnetic field and chemical potential. Right column: The corresponding transverse (imaginary) susceptibilities. For all figures, the quark mass is $1.4 m_{0}=1.54 \mathrm{GeV}$.

susceptibility, calculated from equations (13) and (14), respectively. The longitudinal susceptibility diverges at the deconfinement critical point for all the cases. It is also discontinuous at the first-order transition and has a finite peak in the crossover region. (The discontinuities are difficult to see due to the choice of the figure scale.) The transverse susceptibility is also discontinuous for the first-order transition but does not diverge at the critical point. However, for the finite chemical potential, it develops a cusp and hence is sensitive to the critical point in this case (see the middle and lower right panels of Fig. 2). The $R_{T}$ ratio is shown in Figure 3, where the upper left panel corresponds to the vanishing chemical potential and finite magnetic field, upper right panel to finite chemical potential and vanishing magnetic field and the lower panel shows the simultaneous contribution of finite chemical potential and magnetic field. In all cases, the $R_{T}$ ratio is sensitive to the deconfinement and explicit $Z_{3}$ symmetry breaking. The latter was already indicated in reference [20].

Finally, we consider heavy quark and anti-quark entropies, which can be seen in the right column of Figure 1. When chemical potential vanishes, both entropies are 

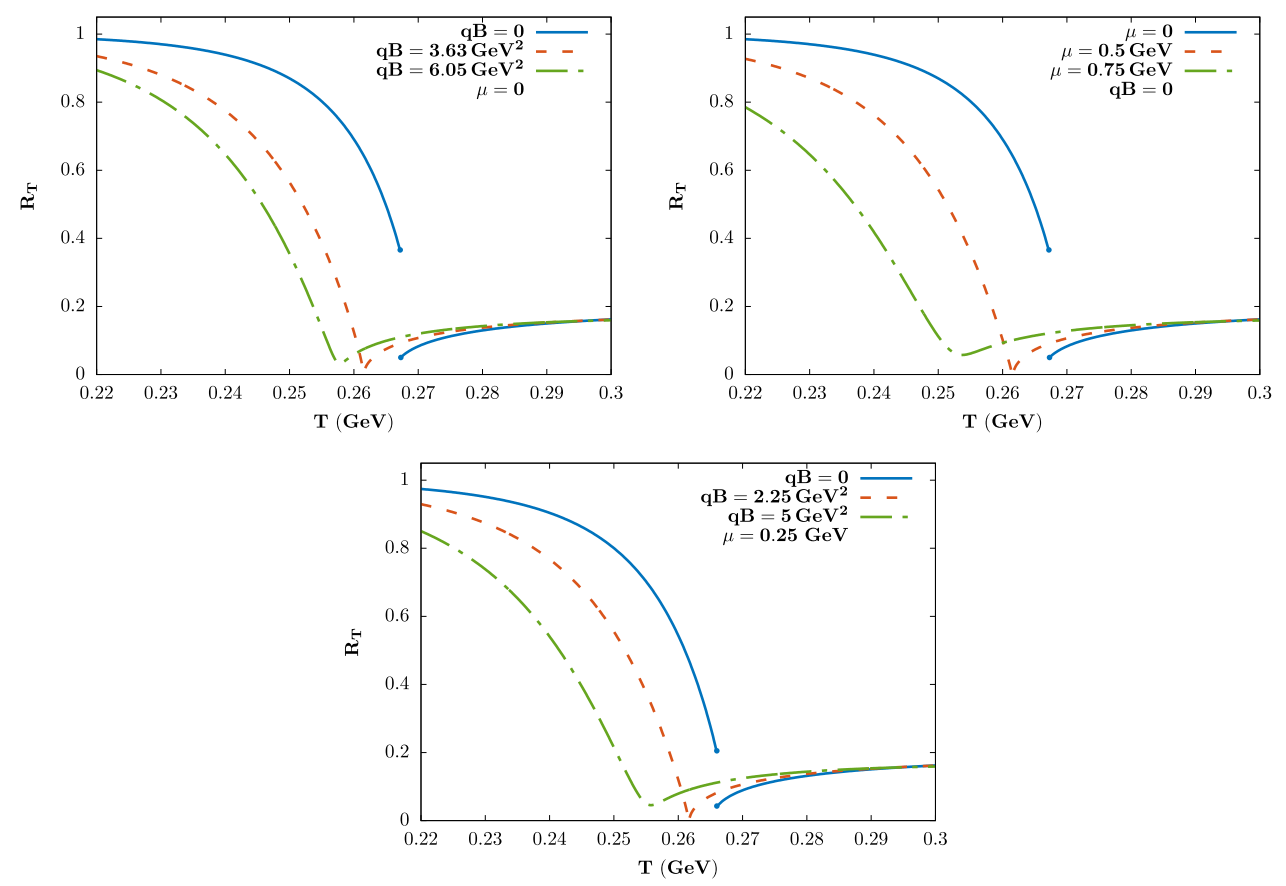

Fig. 3. The $R_{T}$ ratio calculated for different values of chemical potential and external magnetic field. For all figures, the quark mass is $1.4 m_{0}=1.54 \mathrm{GeV}$.

equal. These observables show discontinuities for the first-order transition and diverge at the critical point. The finite peak in case of the crossover provides another way to define a pseudo-critical temperature of deconfinement. When the chemical potential is non-zero, the heavy quark and anti-quark entropies are different, which can be seen in the middle and lower panels. This follows from the fact that expectation values of the Polyakov loop and its conjugate are different. In the first-order region and at the critical point both entropies indicate the same phase transition temperature. In case of the crossover, the peaks are slightly shifted and the maximum of the heavy-quark entropy is at a lower temperature than the maximum of the heavy anti-quark entropy.

\section{Conclusions}

In this work, we discussed the impact of finite chemical potential and external magnetic field on deconfinement in the limit of heavy quarks. We calculated the Polyakov loop, its real and imaginary susceptibilities as well as their ratio. These observables are sensitive to deconfinement. In general, the longitudinal susceptibility diverges at the critical point. On the other hand, the transverse susceptibility remains finite. However, when $\mu$ is greater than 0 this quantity develops a cusp and hence becomes sensitive to the critical point. Additionally, we calculated the heavy quark and anti-quark entropies, which are also sensitive probes of deconfinement, diverging at the critical point. When the chemical potential is non-zero, these quantities are different and their maxima in the crossover region are located at slightly different temperatures.

For heavy quarks the effect of the external magnetic field and finite chemical potential on deconfinement is similar - both quantities enhance the explicit breaking 
of the center symmetry. The crucial difference between these parameters is that the magnetic field does not induce the splitting between the Polyakov loop and its conjugate, while the chemical potential makes these observables different. In this work, we neglected the chiral aspects of QCD. These become important when light quarks are considered, which is relevant for the realistic applications, such as relativistic heavyion collisions and neutron star physics. Further study on these issues will be reported in future works.

The author acknowledges helpful comments and fruitful discussions with Pok Man Lo, Michał Marczenko, Krzysztof Redlich and Chihiro Sasaki. This work was partly supported by the Polish National Science Center (NCN), under Opus Grant No. 2018/31/B/ST2/01663.

Open Access This is an open access article distributed under the terms of the Creative Commons Attribution License (https://creativecommons.org/licenses/by/4.0), which permits unrestricted use, distribution, and reproduction in any medium, provided the original work is properly cited.

Publisher's Note The EPJ Publishers remain neutral with regard to jurisdictional claims in published maps and institutional affiliations.

\section{References}

1. P. Braun-Munzinger, V. Koch, T. Schäfer, J. Stachel, Phys. Rep. 621, 76 (2016)

2. K. Fukushima, T. Hatsuda, Rept. Prog. Phys. 74, 014001 (2011)

3. J.O. Andersen, W.R. Naylor, A. Tranberg, Rev. Mod. Phys. 88, 025001 (2016)

4. J. Greensite, Prog. Part. Nucl. Phys. 51, 1 (2003)

5. E.S. Swanson, AIP Conf. Proc. 717, 636 (2004)

6. A.M. Polyakov, Phys. Lett. 72B, 477 (1978)

7. G.'t Hooft, Nucl. Phys. B 138, 1 (1978)

8. B. Svetitsky, L.G. Yaffe, Nucl. Phys. B 210, 423 (1982)

9. K. Fukushima, V. Skokov, Prog. Part. Nucl. Phys. 96, 154 (2017)

10. C. Allton, S. Ejiri, S. Hands, O. Kaczmarek, F. Karsch, E. Laermann, C. Schmidt, L. Scorzato, Phys. Rev. D 66, 074507 (2002)

11. J. Langelage, O. Philipsen, JHEP 01, 089 (2010)

12. S. Ejiri et al. [WHOT-QCD], PoS Lattice 2012, 089 (2012)

13. P.M. Lo, B. Friman, K. Redlich, Phys. Rev. D 90, 074035 (2014)

14. F. Bruckmann, G. Endrodi, T.G. Kovacs, JHEP 1304, 112 (2013)

15. M. D'Elia, F. Manigrasso, F. Negro, F. Sanfilippo, Phys. Rev. D 98, 054509 (2018)

16. P.M. Lo, K. Redlich, M. Szymański, C. Sasaki, Phys. Rev. D 102, 034024 (2020)

17. P.M. Lo, B. Friman, O. Kaczmarek, K. Redlich, C. Sasaki, Phys. Rev. D 88, 014506 (2013)

18. P.M. Lo, B. Friman, O. Kaczmarek, K. Redlich, C. Sasaki, Phys. Rev. D 88, 074502 (2013)

19. A. Bazavov et al., Phys. Rev. D 93, 114502 (2016)

20. P.M. Lo, M. Szymański, K. Redlich, C. Sasaki, Phys. Rev. D 97, 114006 (2018)

21. K. Binder, Rep. Prog. Phys. 50, 783 (1987)

22. C. Sasaki, B. Friman, K. Redlich, Phys. Rev. D 75, 074013 (2007)

23. A. Dumitru, R.D. Pisarski, D. Zschiesche, Phys. Rev. D 72, 065008 (2005) 